Research Article

\title{
Evaluation of Different Coronal Sealing Materials in the Endodontically Treated Teeth: An In Vitro Study
}

\author{
Tareq Hajaj $\mathbb{D}^{1,2}$ Antonis Perdiou ${ }^{1}{ }^{3},{ }^{3}$ Cosmin Sinescu, ${ }^{1,2}$ Mihai Rominu, ${ }^{1,2}$ \\ Meda Lavinia Negrutiu $\left(\mathbb{0},{ }^{1,2}\right.$ Roxana Talpos, ${ }^{4}$ Adrian Neagu, ${ }^{5}$ and Serban Talpos ${ }^{6}$ \\ ${ }^{1}$ Victor Babes University of Medicine and Pharmacy, Faculty of Dentistry, Department of Propaedeutics and Dental Materials, \\ 2 Eftimie Murgu Sq, Timisoara 300041, Romania \\ ${ }^{2}$ Research Center in Dental Medicine Using Conventional and Alternative Technologies, Faculty of Dental Medicine, \\ Victor Babes University of Medicine and Pharmacy of Timisoara, 9 Revolutiei 1989 Ave, Timisoara 300070, Romania \\ ${ }^{3}$ Victor Babes University of Medicine and Pharmacy, Faculty of Dentistry, Department of Dental Prevention and Oral Health, \\ 2 Eftimie Murgu Sq., Timisoara 300041, Romania \\ ${ }^{4}$ Victor Babes University of Medicine and Pharmacy, Faculty of Dentistry, Department of Endodontics, 2 Eftimie Murgu Sq., \\ Timisoara 300041, Romania \\ ${ }^{5}$ Victor Babes University of Medicine and Pharmacy, Faculty of Medicine, Department of Biophysics, 2 Eftimie Murgu Sq., \\ Timisoara 300041, Romania \\ ${ }^{6}$ Victor Babes University of Medicine and Pharmacy, Faculty of Dentistry, Department of Oral and Maxillofacial Surgery, \\ 2 Eftimie Murgu Sq., Timisoara 300041, Romania
}

Correspondence should be addressed to Antonis Perdiou; aperdiou@gmail.com

Received 5 March 2021; Revised 29 March 2021; Accepted 8 April 2021; Published 21 April 2021

Academic Editor: Ştefan Ţălu

Copyright (C) 2021 Tareq Hajaj et al. This is an open access article distributed under the Creative Commons Attribution License, which permits unrestricted use, distribution, and reproduction in any medium, provided the original work is properly cited.

In vitro studies have shown that rapid penetration of bacteria in the entire root canal system may occur after endodontic treatment without a coronal seal. A proper restorative technique is necessary to ensure a coronal seal and protection of the residual dental structure. The aim of this in vitro study was to evaluate the coronal sealing ability of the most relevant clinical materials by means of dye penetration (neutral red dye, Sigma-Aldrich, Germany), through a light spectrometric device, and to establish which one of the tested dental materials possesses the best sealing ability. Forty-two extracted teeth were prepared and used for this experiment; they were sealed with 5 different cements. The flow composite had the best absorbance value with $0.00675 \pm 0.00096$ (mean \pm standard deviation) for monoradicular samples and $0.025 \pm 0.00129$ for pluriradicular samples. Under the constraints of the present study, both flowable and packable composite materials can be recommended as orifice sealing materials to prevent microleakage in an endodontically treated tooth. To assess the clinical superiority of any material, further in vivo studies are required.

\section{Introduction}

A correct and conclusive diagnosis is of paramount importance when determining whether a tooth needs root canal treatment or not. The clinical protocols have gone a long way, endodontic therapy being one of the most predictable today.

With a success rate of $87.5 \%$ and $88.9 \%$ according to one study, for single visit treatment and $87.4 \%$ according to another $[1,2]$, endodontic therapy is one of the most effective and frequently applied procedures in a dental office.
Clinical symptoms and conditions that might lead to the need of endodontic treatment include unprovoked dental pain, referred or localized pain, especially during postural changes, and prolonged recovery from hot or cold stimuli even after the agent has been removed [3]. Initial root canal treatment is indicated when the pulp is inflamed and the process of capillary collapse has occurred. This process happens due to the increased internal tooth pressure, which is caused by the increased blood supply to the inflamed pulp [4]. This increased blood flow into a nonexpanding space (the pulp chamber) causes capillaries to collapse and 
effectively cut off the blood supply to the pulpal tissues, leading to necrosis [5], which in turn favors bacterial accumulation.

The main culprits responsible for the need for root canal treatment are deep decay, secondary/recurrent caries, repeated dental procedures on a tooth, infected root canals, periapical pathologies, and/or large fillings that are too close to the pulp chamber, as well as a crack or chip in the tooth that is in close proximity to the pulp chamber [6]

The main steps in a successful root canal treatment are accessing the pulp chamber without iatrogenic incidents, establishing a correct working length, and shaping and cleaning the root canal. Working length determination and correct obturating technique are important in order to avoid underfilling or overfilling; the first leaves room for the accumulation of bacteria in the root canal, whereas the second causes inflammation of periapical tissues. During shaping and cleaning, it is very important to irrigate the root canal generously to eliminate all the remaining pulp tissue.

The success of endodontic therapy is broadly thought of in terms of decontamination and complete obturation of the root canal system [7]. The usual factors responsible for an endodontic failure are improper coronal sealing (microleakage), the persistence of bacteria, inside or outside the root canal, underfilling of the canal, unshaped canals (both accessory and major ones), and iatrogenic errors (i.e., poor access cavity preparation and broken instruments) [8].

Another very important part that follows the endodontic filling is the sealing of the pulp chamber and the restoration of the tooth. Regarding this issue, many materials and methods, direct and indirect, have been used over the time, but there is no consensus at this time about which is best. Usually, the type of restoration depends on the remaining tooth structure available and its position on the dental arch, trying to preserve as much hard tissue as possible. In anterior teeth that are minimally to moderately restored, a direct composite restoration will be the material of choice, while a crown will better protect endodontically treated posterior teeth [9]. Other materials and techniques used for the longterm postendodontic restoration include amalgam restorations, gold and ceramic onlays, and ceramic full/partial crowns [9].

As mentioned previously, endodontic treatment has a high rate of success but, interestingly enough, recent studies suggest that even when failures occur; in the majority of cases, it is because of the restoration, not the root canal treatment per se [10]. One of the most common problems clinicians face in today's practice is the phenomenon of microleakage, which seems to affect most, if not all, restorative materials.

Microleakage is defined as the "diffusion of the bacteria, oral fluids, ions, and molecules into the tooth and the filling material interface" or "defined as the clinically undetectable passage of bacteria, fluids, molecules, or ions between tooth and the restorative or filling material" [11]. Clinical investigations, both in vitro and in vivo, have shown that postendodontic coronal microleakage can allow bacterial penetration in the filled root canal system, causing recontamination and failure of the root canal treatment [8]. The success of endodontic therapy hinges on an adequate coronal seal. A well-sealed coronal restoration is essential after the completion of obturation as it will prevent the ingress of microorganisms present in the oral environment [8].

Currently, there are many options of dental materials that are suited for coronal sealing, ranging from glassionomer cements, modified glass-ionomer cements, packable and flowable composites, and all the variations that each company introduces to the market.

Many studies emphasize that tooth filling materials are not inert and impenetrable borders, but dynamic microcrevices, which allow for the busy traffic of bacteria, ions, and molecules which leads to secondary caries [11-13]. The main causes of coronal microleakage of endodontically treated teeth are polymerization shrinkage when bulk filling the cavity, the use of poorly adaptable temporary filling material, and an inadequate marginal adaptation of restorative materials or fixed crowns $[14,15]$. So, the limit between the restoration and dental hard tissue continues to be a zone of clinical concern. It can result in bacterial infiltration and recontamination of the root canal, eventually leading to the failure of the endodontic treatment. Most vulnerable areas of large composite restorations are cuspal areas exposed to large masticatory forces and proximal areas where proper oral hygiene is hard to obtain. In vitro studies have shown that rapid penetration of bacteria of the entire root canal system may occur after endodontic treatment without a coronal seal over the root filling as well as the leakage of an intracanal medicament due to improper coronal sealing [16].

After endodontic treatment, as well as during the treatment, a proper restorative technique is necessary to ensure coronal seal and protection of the residual dental structure $[17,18]$. Hence, it is important to assess the coronal microleakage in the presence of various permanent restorative materials.

To our best knowledge, glass-ionomer cement (GIC), modified glass-ionomer cement (mGIC), and packable and flowable composites, some of the most frequently used restorative materials in the daily practice have never been compared to each other regarding their coronal sealing performance. Therefore, the aim of this study is to evaluate the coronal sealing ability of the most relevant clinical materials mentioned above. To this end, this work proposes a micromolecular dye (neutral red dye, Sigma-Aldrich, Germany) penetration protocol, which employs spectrophotometry to establish which of the tested dental materials possess the best sealing ability.

\section{Materials and Methods}

42 mature extracted human teeth were selected for this study. The selected teeth were stored in formalin for an average of 2 months until the samples were prepared. The selected group contained 21 monoradicular teeth and 21 pluriradicular teeth. In this study, 40 teeth were used to form the 5 experimental groups (see Figures 1 and 2). The remaining two teeth, one from each group, were designated as control samples; these were in pristine condition and no work was done to them besides cleaning and disinfection. 


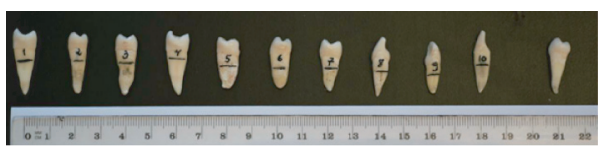

Figure 1: Sample selection of monoradicular teeth and control group after cleaning and disinfection.

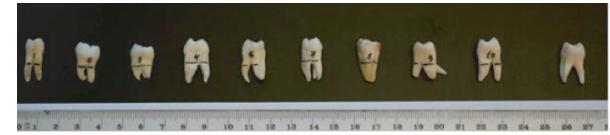

Figure 2: Sample selection of pluriradicular teeth and control group after cleaning and disinfection.

All the selected teeth had four intact coronal walls and normal root morphology. After careful clinical examination, teeth with fractures, cracks, caries involving the coronal part or the root, previously endodontically treated teeth, and teeth with complicated root morphology were excluded from the study. The teeth were cleaned of the biological debris by hand and then were thoroughly cleaned with an ultrasonic scaler. Disinfection was done with Chloraxid 2\% (sodium hypochlorite 2\%, Cerkamed, Poland) and GlucoChex 2\% (chlorhexidine digluconate 2\%, Cerkamed, Poland) for 1 hour each and then stored in physiologic saline solution until use $[19,20]$. The preparatory procedures for all the extracted teeth were standardized for all groups and were performed by a single operator. Coronal access was assured and working lengths were selected at $1 \mathrm{~mm}$ short of the point at which a number $10 \mathrm{~K}$-file exited the apical foramen. Coronal flaring was accomplished by the gates Glidden burs, sizes 2 and 3. The canals were instrumented with $\mathrm{K}$-files (Dentsply Maillefer, Switzerland). ENDO-Prep Cream with $15 \%$ EDTA and $10 \%$ urea peroxide (Cerkamed, Poland) was used to coat the K-files while they were used. Between successive files, the root canals were irrigated with 5.25\% sodium hypochlorite (Cerkamed, Poland) and physiologic saline using an endoneedle alternatively. Throughout the cleaning and shaping procedures, apical patency was maintained by recapitulation with a number $10 \mathrm{~K}$-file. All teeth were instrumented in the same manner. The smear layer was removed using $2 \mathrm{ml}$ of $15 \%$ EDTA solution (Cerkamed, Poland) followed by a final flush with $2 \mathrm{ml}$ of Chloraxid 2\% (sodium hypochlorite 2\%, Cerkamed, Poland). Upon completion of the instrumentation, the canals were dried utilizing absorbent paper points. This process was done to ensure that all canals and especially the point of interest, the canal orifices, were conditioned and prepared with the same working protocol, thereby assuring homogenous conditions for this study. Since the main objective of this research is the coronal microleakage at the level of canal orifices, the experimental teeth were purposefully not obturated with any materials, giving us more room for the neutral red dye. Because of the vast assortment of commercially available materials, for this study, we used the most relevant clinical options. The following materials were investigated: Kavitan Plus, glass-ionomer cement, GIC (PENTRON, Orange, USA); Ionosit, light cured compomer,

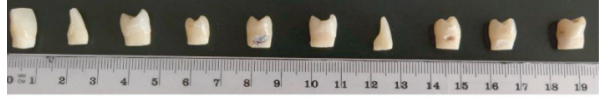

Figure 3: Monoradicular teeth cut 2-5 mm from CEJ.

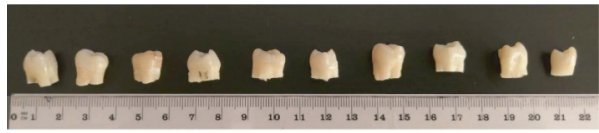

Figure 4: Pluriradicular teeth cut 2-5 mm from CEJ.

mGIC (DMG, Hamburg, Germany); brilliant flow, flowable composite (COLTENE, Altstätten, Switzerland); Kerr Premise, packable composite (KERR, Brea, USA); Coltosol F, temporary filling material (COLTENE, Altstätten, Switzerland). Both composites used are made of nanoparticles. The roots of the 20 teeth in the experimental group were cut short $2-5 \mathrm{~mm}$ (depending on the pulp chamber morphology) from the level of cementoenamel junction (CEJ) with a tapered fissured diamond bur in a high-speed handpiece under water spray (Figures 3 and 4). This was done for two main reasons: to have enough space for the neutral red dye, without the dye freely flowing through the whole root canal and potential leak through lateral or accessory canals, and to ensure a hermetic seal of the roots where they were sealed apically up to the CEJ with flowable composite.

The teeth employed in this study were divided into six groups. Groups I to V were experimental groups and Group VI was the control group. Each experimental group comprised four monoradicular and four pluriradicular teeth, whereas the control group was composed of one monoradicular tooth and one pluriradicular tooth. The purpose of the control group was to establish potential discoloration and other changes in the saline solution when the pristine tooth was submerged in physiological saline for the whole duration of the experiment. Each experimental group was tested in a separate sterile container made of hard plastic, with a total volume of $25 \mathrm{~mL}$, and with a screw lock cap; they were purchased from a local pharmacy. In order to have all the teeth fully submerged in the same amount of physiological saline solution, we measured the volume of solution needed to cover the largest tooth: the pluriradicular tooth from the control group (a molar). The same volume, $10 \mathrm{~mL}$, was used for all the samples. First, we applied a hermetic seal to all the teeth, experimental and control group, using Premise Flowable Light Cure Composite (KERR, Brea, USA) together with OptiBond Solo Plus bonding and etching (37.5\% phosphoric acid) system (KERR, Brea, USA). The teeth were dried, etched up to the CEJ, for 15 seconds, and rinsed vigorously for an additional 30 seconds (to ensure that all etching was removed). After drying lightly, the bonding was applied to all the areas up to the CEJ. After light curing for 20 seconds, the flowable composite was placed and light cured for 40 seconds. The remaining two teeth (one monoradicular and one pluriradicular) were hermetically sealed up to the CEJ and designated as control for each group. These two teeth were in pristine condition and no work was done on them, except for cleaning and disinfection. The role the hermetic 
seal applied to these particular teeth was to ensure a tight entrapment of the root canal contents. Once the hermetic seal was established, the neutral red dye was placed into the root canal. Endodontic microsponges (LARIDENT, Italy) were used to absorb and contain the neutral red dye inside the root canal and to assure enough firmness to apply the orifice sealing material on top of it, without the materials forcing the dye out of the root canal and into the pulp chamber. The endodontic microsponges were cut into small pieces of about $2 \times 2 \mathrm{~mm}$ to $4 \times 4 \mathrm{~mm}$ to accommodate different pulp chamber sizes. With the help of a plastic pipette, one drop of neutral red dye was applied inside the root canal of the experimental teeth and the orifice was covered with a piece of microsponge. The orifice sealing material, according to each testing group, was applied on top of the sponge to create an initial barrier and afterward the bulk of the testing orifice sealing material was applied. After orifice sealing, the teeth were submerged in $10 \mathrm{~mL}$ physiological saline solution in individual plastic containers. The containers were shaken for 30 seconds, 2 times per day for 2 weeks. After 2 weeks, a sample of the saline solution was taken from each container and was analysed with the help of a Metertech SP-880 UV/ visible spectrophotometer (Metertech, Taipei, Taiwan). Before the analysis of the samples, the peak absorbance point of the tested dye solution was obtained. The peak absorbance point was established at the $540 \mathrm{~nm}$. The saline solution from the container was used for base line (zero absorbance) calibration and the saline solution with one drop of Neutral red dye was used to assess the wavelength of maximum absorbance. As a result, we obtained the peak absorbance point at $540 \mathrm{~nm}$. Finally, the solution from each experimental group was tested (i.e., its absorbance was measured at a wavelength of $540 \mathrm{~nm}$ ) and the data were recorded. The higher the resulting absorbance value, the weaker the orifice sealing will be.

\section{Results}

All the experimental groups showed some level of dye leakage into the saline solution. In general, monoradicular teeth displayed less leakage. Table 1 presents the absorbance values recorded for Group I, composed of 4 teeth with orifice sealing performed with a temporary cement, Coltosol $\mathrm{F}$ (COLTENE, Altstätten, Switzerland).

Absorbance values recorded in this study lie between 0 (the "blank" value, obtained for unspoiled saline solution) and the maximum value of 0.055 (obtained during the measurement of the peak absorbance when one dye drop was uniformly distributed in $10 \mathrm{~mL}$ saline solution, see previous section). Lower numbers indicate better orifice sealing capabilities. Absorbance results for the other 4 materials investigated in this work are shown in Table 2.

A comparison of the results listed in Tables 1 and 2 leads to the unsurprising conclusion that a temporary cement assures weaker orifice sealing than permanent restoration materials. The mean values of the absorbance values obtained for monoradicular and pluriradicular teeth are shown in Figures 5 and 6, respectively, along with the corresponding standard deviations (SD) depicted as vertical error bars.
TABLE 1: Absorbance values obtained for the samples prepared using the Coltosol temporary cement.

\begin{tabular}{lc}
\hline Sample & Absorption at $540 \mathrm{~nm}$ \\
\hline $1^{\text {st }}$ monoradicular sample & 0.025 \\
$2^{\text {nd }}$ monoradicular sample & 0.024 \\
$3^{\text {rd }}$ monoradicular sample & 0.026 \\
$4^{\text {th }}$ monoradicular sample & 0.025 \\
$1^{\text {st }}$ pluriradicular sample & 0.052 \\
$2^{\text {nd }}$ pluriradicular sample & 0.054 \\
$3^{\text {rd }}$ pluriradicular sample & 0.051 \\
$4^{\text {th }}$ pluriradicular sample & 0.052 \\
\hline
\end{tabular}

The mean absorbance value for Coltosol $\mathrm{F}$ for monoradicular samples was 0.025 with SD 0.00082 , and for pluriradicular samples, the mean value was 0.05225 with SD 0.00126 . For GIC, the mean value for monoradicular samples was 0.016 with SD 0.00141 , whereas for pluriradicular samples, it was 0.03875 with SD 0.00096 . For mGIC, we obtained a mean value of 0.014 with SD 0.00082 for monoradicular samples and a mean 0.02725 with SD 0.0025 for pluriradicular samples. The brilliant flow had the best mean absorbance value, 0.00675 , with SD 0.00096 for monoradicular samples and the best mean absorbance value, 0.0205 with SD 0.00129 , for pluriradicular samples. The packable composite Kerr Premise had a mean absorbance value of 0.011 with SD 0.00082 for monoradicular samples and a mean absorbance value of 0.02375 with SD 0.00171 for pluriradicular samples.

\section{Discussion}

As seen from the results, there are big differences between restorative materials. This is a clear sign that the immediate crown restoration of the endodontically treated tooth is vital to the final outcome of the therapy. The disinfection and filling of the root canal may be mandatory, but they are not irreversible; at any time, due to a lack of substance or poorly adapting restoration, the oral bacteria could easily recolonize the pulp canal. By that point, it is only a matter of time until the infectious process restarts. Also, it is very important for the clinicians not to overlook the provisional restoration, which is very common after root canal treatments. There is no given time that should be "safe" for the tooth to be restored with a material that does not meet the highest standards regarding microleakage, such as temporary cements. It becomes clear that even for short-term provisional restoration, the material of choice should be a light cured flow composite.

A meta-analysis conducted by $\mathrm{Ng}$ et al., as well as another retrospective study including 1001 endodontically treated teeth, indicates that the success rate of endodontically treated teeth was higher if they had a good quality coronal restoration [21, 22].

To prevent loss of the crucial coronal seal, a restoration is needed after the endodontic treatment is completed. This postendodontic restoration should be able to prevent the recontamination of the root canal system by keeping it free from food debris, oral fluids, and microorganisms present in 
TABLE 2: Absorbance values obtained for all permanent cements.

\begin{tabular}{lcccccccc}
\hline $\begin{array}{l}\text { Permanent } \\
\text { cement }\end{array}$ & $\begin{array}{c}1 \text { st } \\
\text { monoradicular }\end{array}$ & $\begin{array}{c}2^{\text {nd }} \\
\text { monoradicular }\end{array}$ & $\begin{array}{c}3^{\text {rd }} \\
\text { monoradicular }\end{array}$ & $\begin{array}{c}4^{\text {th }} \\
\text { monoradicular }\end{array}$ & $\begin{array}{c}1^{\text {st }} \\
\text { pluriradicular }\end{array}$ & $2^{\text {nd }}$ & $3^{\text {pd }}$ & $4^{\text {th }}$ \\
GIC & 0.014 & 0.017 & 0.016 & 0.017 & 0.038 & 0.040 & 0.038 \\
mGIC & 0.013 & 0.014 & 0.014 & 0.015 & 0.024 & 0.030 & 0.028 \\
$\begin{array}{l}\text { Flow } \\
\text { composite }\end{array}$ & 0.006 & 0.007 & 0.008 & 0.006 & 0.019 & 0.021 & 0.020 & 0.027 \\
$\begin{array}{l}\text { Packable } \\
\text { composite }\end{array}$ & 0.010 & 0.012 & 0.011 & 0.011 & 0.022 & 0.024 & 0.026 \\
\hline
\end{tabular}

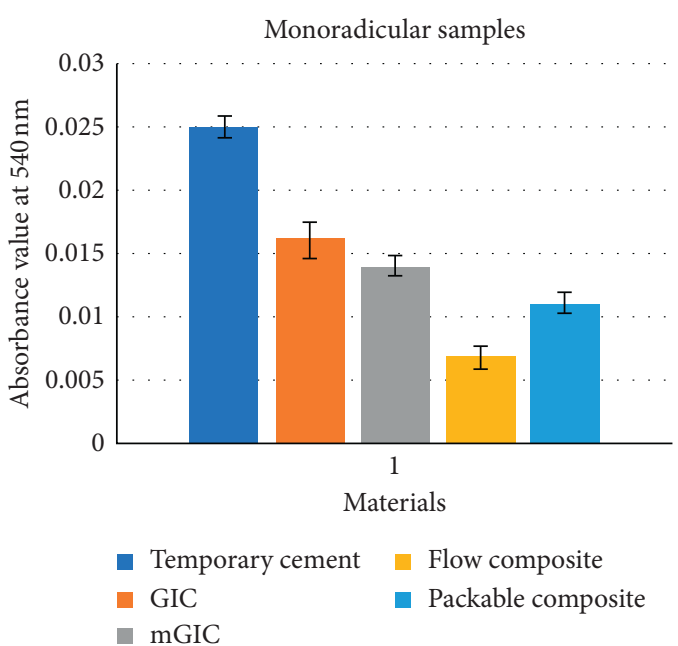

Figure 5: Mean value for all materials for monoradicular samples.

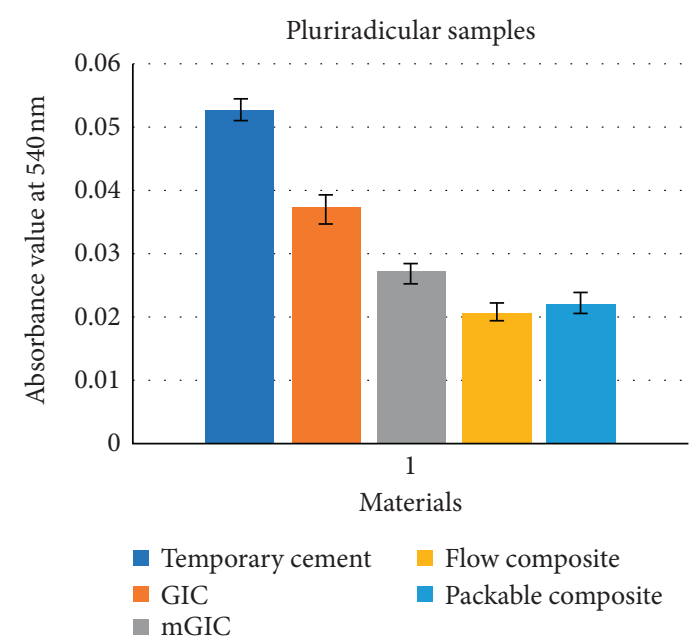

Figure 6: Mean value for all materials for pluriradicular samples.

the oral cavity [12]. Currently, several dental materials are used to achieve a coronal seal and each of them has its own benefits and limitations. To our knowledge, there are few comparative studies aiming to assess the above materials as orifice sealants. Divya et al. published an in vitro study on a sample of 70 extracted human teeth, comparing the sealing capability of light cured composite resin, GMTA (gray mineral trioxide aggregate), WMTA (white mineral trioxide aggregate), and GIC type II. Their results were similar to this study showing that composite resin had better sealing abilities than GIC [23]. Gerdolle et al. compared the microleakage of a compomer, an ormocer, a packable composite, and a resin modified GIC in another in vitro study performed on a sample of 40 mandibular human teeth. They observed that the resin modified GIC and the packable composite had the best sealing abilities, which was observed in the present study, as well [24].

Mali et al. performed a similar study on 30 extracted teeth, comparing the microleakage of glass ionomer, composite resin, and compomers. The conclusion was that glassionomer cements had the highest rate of microleakage, while the composite came second and the compomer had the best results [25]. In a clinical environment, there are several factors that can potentially influence the quality of the final work, from the quality of cavity preparation to the damage extent of coronal tissues. Composite microleakage can be attributed to the $\mathrm{C}$-factor or configuration factor, which refers to the ratio of bonded to unbonded surfaces in cavity, which may increase the polymerization shrinkage [26]. As the $\mathrm{C}$-factor increases, the compensation for polymerization shrinkage by the flow of composite decreases, and thus, the polymerization stress at the bonded surface increases. Also, even within the composite restorations' category, there are different protocols meant to overcome the problem of microleakage. One of the most revealing studies was done on 60 extracted teeth and found out that rebonding with a surface sealant can reduce marginal microleakage of composite resin restorations better than rebonding with a bonding agent [27]. The methodology proposed in this work is quantitative, reproducible, and adaptable for different studies of dental microleakage. Although the results reported here are in agreement with the published literature, this study has limitations that prevent the extrapolation of the results to the clinical context. First, it is an in vitro study performed on a relatively low number of extracted teeth. Second, it investigates the ability of dental materials to prevent the infiltration of a micromolecular solution (molecular weight of 288.8), which is not an indicative of bacterial infiltration. The present study can be the base of an in vivo, prospective study specifically designed to identify the clinical performance of different sealing materials placed in the complex environment of the oral cavity. Along with the investigations from Kim et.al and Lee et. al $[28,29]$, our results suggest that the use of a double material seal, in conjunction with incremental techniques used for composite delivery, albeit more time consuming, will result in better orifice and coronal sealing than the use of a single coronal sealing material followed by bulk-fill composite technique. 


\section{Conclusion}

On the basis of the results of this study, we can conclude that the flow composite had the best sealing ability followed by the packable composite. Nevertheless, all the materials investigated in this work can be used effectively as sealing materials. Further in vivo studies will be needed to assess the clinical superiority of one material over the other.

\section{Data Availability}

No data were used to support this study.

\section{Conflicts of Interest}

The authors declare that they have no conflicts of interest.

\section{References}

[1] F. Moazami, S. Sahebi, F. Sobhnamayan, and A. Alipour, "Success rate of nonsurgical endodontic treatment of nonvital teeth with variable periradicular lesions," Iranian Endodontic Journal, vol. 6, no. 3, pp. 119-124, 2011.

[2] A. W. Wong, C. S. Tsang, S. Zhang, K. Y. Li, C. Zhang, and C. H. Chu, "Treatment outcomes of single-visit versus multiple-visit non-surgical endodontic therapy: a randomised clinical trial," BMC Oral Health, vol. 15, p. 162, 2015.

[3] K. M. Hargeaves and S. Cohen, Pathways of the Pulp, Mosby Elsevier, Maryland Heights, Missouri, 2011.

[4] K. J. Heyeraas and I. Kvinnsland, "Tissue pressure and blood flow in pulpal inflammation," Journal of Dental Research, vol. 88, no. 1, pp. 393-401, 1992.

[5] K. J. Heyeraas and E. Berggreen, "Interstitial fluid pressure in normal and inflamed pulp," Critical Reviews in Oral Biology \& Medicine, vol. 10, no. 3, pp. 328-336, 1999.

[6] AAE, Reasons for Root Canal Treatment, American Association of Endodontists, Chicago, Illinoi, USA, 2019, http:// www.aae.org/patients/root-canal-treatment/what-is-a-rootcanal/reasons-root-canal-treatment/.

[7] M. Haapasalo, Y. Shen, Z. Wang, and Y. Gao, "Irrigation in endodontics," British Dental Journal, vol. 216, no. 6, pp. 299-303, 2014.

[8] S. Tabassum and F. R. Khan, "Failure of endodontic treatment: the usual suspects," European Journal of Dentistry, vol. 10, no. 01, pp. 144-147, 2016.

[9] F. Mannocci and J. Cowie, "Restoration of endodontically treated teeth," British Dental Journal, vol. 216, no. 6, pp. 341-346, 2014.

[10] Y. Zadik, V. Sandler, R. Bechor, and R. Salehrabi, "Analysis of factors related to extraction of endodontically treated teeth," Oral Surgery, Oral Medicine, Oral Pathology, Oral Radiology, and Endodontology, vol. 106, no. 5, pp. e31-e35, 2008.

[11] S. Muliyar, K. A. Shameem, R. P. Thankachan, P. G. Francis, C. S. Jayapalan, and K. A. Hafiz, "Microleakage in endodontics," Journal of International Oral Health, vol. 6, no. 6, pp. 99-104, 2014.

[12] M. M. Ebaya, A. I. Ali, and S. H. Mahmoud, "Evaluation of marginal adaptation and microleakage of three glass ionomerbased class V restorations: in vitro study," European Journal of Dentistry, vol. 13, no. 04, pp. 599-606, 2019.

[13] T. Rodig and M. Hulsmann, "Restorative materials for the temporary seal of the endodontic access cavity," Journal of Endodontics, vol. 2, no. 2, pp. 117-130, 2008.
[14] A. A. Al Habdan, "Review of microleakage evaluation tools," Journal of International Oral Health, vol. 9, pp. 141-145, 2017.

[15] I. S. Dragland, H. Wellendorf, H. Kopperud, I. Stenhagen, and H. Valen, "Investigation on the antimicrobial activity of chitosan-modified zinc oxide-eugenol cement," Biomaterial Investigations in Dentistry, vol. 6, no. 1, pp. 99-106, 2019.

[16] S. Balaji, K. Kumar, R. Venkatesan, S. Krishnamoorthy, V. Manoharan, and S. Marimuthu, "Assessment of coronal leakage with two intracanal medicaments after exposure to human saliva-an in vitro study," International Journal of Clinical Pediatric Dentistry, vol. 11, no. 5, pp. 406-411, 2018.

[17] A. Libonati, V. Di Taranto, G. Gallusi, E. Montemurro, and V. Campanella, "CAD/CAM customized glass fiber post and core with digital intraoral impression: a case report," Clinical, Cosmetic and Investigational Dentistry, vol. 12, pp. 17-24, 2020.

[18] M. Lalitagauri, "Importance of coronal seal: preventing coronal leakage in endodontics," Journal of Dental Research, vol. 3, no. 4, pp. 71-75, 2016.

[19] J. Marion, F. Manhães, H. Bajo, and T. Duque, "Efficiency of different concentrations of sodium hypochlorite during endodontic treatment," Literature review, vol. 2, pp. 32-37, 2012.

[20] Z. Mohammadi, "Chlorhexidine gluconate, its properties and applications in endodontics," Iranian Endodontic Journal, vol. 2, no. 4, pp. 113-125, 2008.

[21] L. Tronstad, K. Asbjørnsen, L. Døving, I. Pedersen, and H. M. Eriksen, "Influence of coronal restorations on the periapical health of endodontically treated teeth," Dental Traumatology, vol. 16, no. 5, pp. 218-221, 2000.

[22] Y. L. Ng, V. Mann, S. Rahbaran, J. Lewsey, and K. Gulabivala, "Outcome of primary root canal treatment: systematic review of the literature -- Part 2. Influence of clinical factors," International Endodontic Journal, vol. 41, no. 1, pp. 6-31, 2008.

[23] K. T. Divya, G. Satish, T. S. Srinivasa, V. Reddy, K. Umashankar, and B. M. Rao, "Comparative evaluation of sealing ability of four different restorative materials used as coronal sealants: an in vitro study," Journal of International Oral Health, vol. 6, no. 4, pp. 12-17, 2014.

[24] D. A. Gerdolle, E. Mortier, and D. Droz, "Microleakage and polymerization shrinkage of various polymer restorative materials," Journal of Dentistry for Children, vol. 27, 2008.

[25] P. Mali, S. Deshpande, and A. Singh, "Microleakage of restorative materials: an in vitro study," Journal of the Indian Society of Pedodontics and Preventive Dentistry, vol. 24, no. 1, pp. 15-18, 2006.

[26] D. Banomyong, J. E. A. Palamara, H. H. Messer, and M. F. Burrow, "Sealing ability of occlusal resin composite restoration using four restorative procedures," European Journal of Oral Sciences, vol. 116, no. 6, pp. 571-578, 2008.

[27] A. Mariani, G. Sutrisno, and M. Usman, "Marginal microleakage of composite resin restorations with surface sealant and bonding agent application after finishing and polishing," Journal of Physics: Conference Series, vol. 1073, Article ID 042005, 2018.

[28] M. E. Kim and S. H. Park, "Comparison of premolar cuspal deflection in bulk or in incremental composite restoration methods," Operative Dentistry, vol. 36, no. 3, pp. 320-340, 2011.

[29] M.-R. Lee, B.-H. Cho, H.-H. Son, C.-M. Um, and I.-B. Lee, "Influence of cavity dimension and restoration methods on the cusp deflection of premolars in composite restoration," Dental Materials, vol. 23, no. 3, pp. 288-295, 2007. 